\title{
ARITHMETICAL NOTES, I. ON A THEOREM OF VAN DER CORPUT
}

\author{
ECKFORD COHEN
}

1. Introduction. Let $f(n)$ denote the arithmetical integral of the complex-valued arithmetical function $g(n)$,

$$
f(n)=\sum_{d \delta=n} g(d)
$$

We recall the following important corollary of Axer's theorem $[1, \S 1]$ concerning the mean values of arithmetical functions.

Theorem A (cf. Remark 1.1 below). If the series,

$$
\sum_{n=1}^{\infty} \frac{g(n)}{n}
$$

is absoutely convergent, then

$$
\lim _{x \rightarrow \infty}\left(\frac{1}{x} \sum_{n \leqq x} f(n)\right)=\alpha \equiv \sum_{n=1}^{\infty} \frac{g(n)}{n} .
$$

For integers $k \geqq 1$, let $f_{k}(n)$ denote the $k$-tuple arithmetical integral of $g(n)$,

$$
f_{k}(n)=\sum_{d d_{1}, \ldots d_{k}=n} g(d) .
$$

In this note we shall prove the following generalization of Theorem A.

THEOREM B. If the series in (1.2) is absolutely convergent, then for $k \geqq 1$,

$$
\lim _{x \rightarrow \infty}\left(\frac{1}{x \log ^{k-1} x} \sum_{n \leqq x} f_{k}(n)\right)=\alpha_{k} \equiv \frac{\alpha}{(k-1) !} .
$$

Clearly (1.5) reduces to (1.3) in case $k=1$. Van der Corput proved a result equivalent to Theorem $\mathrm{B}$ in the special case in which $g(n)$ is assumed multiplicative [2, Proposition 1]. However, van der Corput did not make explicit use of the multiple arithmetical integral, with the consequence that the essential simplicity of his argument is somewhat obscured. Moreover, the superfluous multiplicativity assumption leads to additional complications in his method.

We shall need two simple lemmas.

Presented to the Society, February 27, 1960 under the title Note on a theorem of van der Corput; received by the editors May 18, 1960. 
Lemma 1.1. For fixed $a \geqq 0$ and all $x \geqq 1$,

$$
\sum_{n \leq x} \frac{\log ^{a} n}{n}=\frac{\log ^{a+1} x}{a+1}+O(1) .
$$

Proof. Since $\left(\log ^{a} x\right) / x$ is monotonically decreasing for $x \geqq e^{a}$, we have $[7$, Theorem 6, p. 10]

$$
\sum_{n \leqq x} \frac{\log ^{a} n}{n}=\int_{1}^{x}\left(\frac{\log ^{a} t}{t}\right) d t+O(1),
$$

from which (1.6) results.

For a natural number $j$, let $\tau_{j}(n)$ denote the number of decompositions of $n$ into a product of $j$ factors, where order of the factors is taken into consideration $\left(\tau_{1}(n) \equiv 1\right)$.

Lemma 1.2. If $j \geqq 1$, then

$$
T_{j}(x) \equiv \sum_{n \leq x} \tau_{j}(n)=\left\{\begin{array}{lr}
O\left(x \log ^{j-1} x\right) & (x \geqq 2) \\
O(1) & (1 \leqq x<2) .
\end{array}\right.
$$

Proof. The lemma is obvious in case $j=1$. Arguing inductively, suppose that $i>1$ and assume the lemma in case $j=i-1$. On the basis of (1.6) with $a=0$, one obtains for $x \geqq 2$,

$$
\begin{aligned}
T_{i}(x) & =\sum_{n \leq x} \tau_{i}(n)=\sum_{d_{1} \cdots d_{i} \leq x} 1=\sum_{n \leq x} T_{i-1}\left(\frac{x}{n}\right) \\
& =\sum_{n \leq x / 2} O\left(\frac{x}{n} \log ^{i-2} \frac{x}{n}\right)+O(x)=O\left(x \log ^{i-2} x \sum_{n \leq x} \frac{1}{n}\right) \\
& =O\left(x \log ^{i-1} x\right),
\end{aligned}
$$

thus completing the induction.

REMARK 1.1. For a simple independent proof of Theorem A, see van der Corput [2, Proposition 4]. That proof is based on the following result of Kronecker [4, Theorem 3, $\left(p_{n}=n\right)$, p. 129;5, §3], appearing in van der Corput as [2, Lemma 1]: If (1.2) converges, then $\sum_{n \leqq x} g(n)$ $=o(x)$.

Applications of Theorem A are contained in [1, §3].

2. Proof of Theorem B. We proceed by induction on $k$. Since the theorem is true in case $k=1$ (Theorem $\mathrm{A}$ ), we shall suppose $k=r>1$ in what follows, assuming the validity of Theorem $\mathrm{B}$ for $k=r-1$.

Place

$$
f_{r}(n)=\sum_{d \delta=n} h_{r}(d), \quad h_{r}(n)=\sum_{d d_{1}} \cdots d_{r-1=n} g(d) .
$$


Let $H_{r}(x)$ and $H_{r}^{*}(x)$ denote, respectively, the summatory functions of $h_{r}(n)$ and $\left|h_{r}(n)\right|$. The induction hypothesis applied to $h_{r}(n)$ asserts that as $x \rightarrow \infty$,

$$
H_{r}(x) \equiv \sum_{n \leq x} h_{r}(n)=\alpha_{r-1} x \log ^{r-2} x+o\left(x \log ^{r-2} x\right) .
$$

We have from (2.1), supposing $x \geqq 2$,

$$
\sum_{n \leq x} f_{r}(n)=\sum_{d \delta \leq x} h_{r}(d)=\sum_{n \leq x} h_{r}(n)\left[\frac{x}{n}\right]
$$

so that

$$
F_{r}(x) \equiv \sum_{n \leq x} f_{r}(n)=x \sum_{n \leq x} \frac{h_{r}(n)}{n}+O\left(H_{r}^{*}(x)\right) .
$$

By partial summation and (2.2) (cf. [6, p. 11, 70,s=0]),

$$
\begin{aligned}
\sum_{n \leq x} \frac{h_{r}(n)}{n} & =\sum_{n \leq x} \frac{B_{r}(n)}{n(n+1)}+\frac{B_{r}(x)}{[x]+1} \\
& =\alpha_{r-1} \sum_{n \leq x} \frac{\log ^{r-2} n}{n}+o\left(\sum_{n \leq x} \frac{\log ^{r-2} n}{n}\right)+O\left(\log ^{r-2} x\right) ;
\end{aligned}
$$

hence by Lemma 1.1,

$$
\sum_{n \leq x} \frac{h_{r}(n)}{n}=\alpha_{r} \log ^{r-1} x+o\left(\log ^{r-1} x\right) .
$$

We also have by (2.1), using Lemma 1.2,

$$
\begin{aligned}
H_{r}^{*}(x) & \equiv \sum_{n \leq x}\left|h_{r}(n)\right| \leqq \sum_{d d_{1}, \ldots d_{r-1 \leq x}}|g(d)|=\sum_{n \leq x}|g(n)| T_{r-1}\left(\frac{x}{n}\right) \\
& =O\left(x \sum_{n \leq x / 2} \frac{|g(n)|}{n} \log ^{r-2} \frac{x}{n}\right)+O\left(\sum_{n \leq x}|g(n)|\right) .
\end{aligned}
$$

By partial summation and the absolute convergence of (1.2), the second $O$-term is seen to be $O(x)$. Hence one obtains, again using the absolute convergence of (1.2),

$$
H_{r}^{*}(x)=O\left(x \log ^{-2} x \sum_{n \leq x} \frac{|g(n)|}{n}\right)=o\left(x \log ^{r-1} x\right) .
$$

Combining (2.3), (2.4) and (2.5), one concludes that as $x \rightarrow \infty$,

$$
F_{r}(x)=\alpha_{r} x \log ^{r-1} x+o\left(x \log ^{r-1} x\right),
$$


which completes the induction, thus proving the theorem.

3. An example. Suppose that $r$ is a positive integer and let $\tau_{k}^{(r)}(n)$ denote the number of representations of $n$ as a product, $n=d_{1}^{r} d_{2} \cdots$ $d_{k}, \tau_{k}^{(1)}(n)=\tau_{k}(n)$.

THEOREM 3.1. If $r \geqq 1$, then as $x \rightarrow \infty$,

$$
T_{k}^{(r)}(x) \equiv \sum_{n \leq x} \tau_{k}^{(r)}(n) \sim \begin{cases}\frac{x \log ^{k-1} x}{(k-1) !} & \text { if } r=1, k \geqq 1, \\ \frac{\zeta(r) x \log ^{k-2} x}{(k-2) !} & \text { if } r>1, k>1,\end{cases}
$$

where $\zeta(r)$ denotes the Riemann $\zeta$-function.

Proof. Case $1(r=1)$. Define $g(n)$ to be 1 or 0 according as $n=1$ or $n>1$, so that in (1.4), $f_{k}(n)=\tau_{k}(n)$. The first part of (3.1) follows by Theorem B with $\alpha=1$.

Case $2(r>1)$. Place $g(n)=1$ or 0 according as $n$ is or is not an $r$ th power. Then $f_{k-1}(n)=\tau_{k}^{(r)}(n)$ in (1.4), $k>1$. The second part of (3.1) is a consequence of Theorem B with $\alpha=\zeta(r)$, and $k$ replaced by $k-1$.

REMARK 3.1. The first result in (3.1) refines the estimate for $T_{k}(x)$ proved in Lemma 1.2. For an even sharper elementary approximation in this case, we refer to the classical estimate in the Piltz divisor problem [3, p. 291, $s=0]$.

\section{BIBLIOGRAPHY}

1. F. V. Atkinson and Lord Cherwell, The mean-values of arithmetical functions, Quart. J. Math. Oxford Ser. (2) vol. 20 (1949) pp. 65-79.

2. J. G. van der Corput, Sur quelques fonctions arithmetiques elementaires, Proc. Roy. Acad. Sci. (Amsterdam) vol. 42 (1939) pp. 859-866.

3. L. E. Dickson, History of the theory of numbers, Vol. I, reprinted New York, Chelsea, 1952.

4. Konrod Knopp, Theory and application of infinite series, New York (corresponding to 4 th German ed., 1947).

5. Leopold Kronecker, Quelques remarques sur la détermination des valeurs moyennes, Comptes Rendues de l'Académie des Sciences Française vol. 103 (1886), pp. 980-987.

6. G. P6lya and G. Szegö, Aufgaben und Lehrsätze der Analysis, Vol. I, 2d ed., Berlin, Springer-Verlag, 1954.

7. Wilhelm Specht, Elementare Beweise der Primzahlsätze, Berlin, Deutscher Verlag der Wissenschaften, 1956.

The University of Tennessee 